\title{
Photomechanical bending behavior of
}

\section{photochromic diarylethene crystals induced under polarized light}

\section{Akira Hirano, Daichi Kitagawa, Seiya Kobatake}

\begin{tabular}{|c|l|}
\hline Citation & CrystEngComm, 21(15); 2495-2501 \\
\hline Issue Date & 2019-04-21 \\
\hline Type & Journal Article \\
\hline Textversion & Author \\
\hline $\begin{array}{c}\text { Supplementary } \\
\text { files }\end{array}$ & $\begin{array}{l}\text { Supplementary information and movie is available at } \\
\text { https://doi.org/10.1039/C9CE00175A. }\end{array}$ \\
\hline Relation & $\begin{array}{l}\text { The following article has been accepted by CrystEngComm. This is accepted } \\
\text { manuscript. The final, published version is available at } \\
\text { https://doi.org/10.1039/C9CE00175A. }\end{array}$ \\
\hline DOI & 10.1039/C9CE00175A \\
\hline
\end{tabular}

\author{
Self-Archiving by Author(s) \\ Placed on: Osaka City University
}

HIRANO A., KITAGAWA D., \& KOBATAKE S. (2019). Photomechanical bending behavior of photochromic diarylethene crystals induced under polarized light. CrystEngComm. 21, 2495-2501. doi:10.1039/C9CE00175A. 


\section{CrystEngComm}

\section{ARTICLE}

Received 00th January 20xx, Accepted 00th January 20xx

DOI: $10.1039 / \times 0 \times x 00000 x$

\section{Photomechanical bending behavior of photochromic diarylethene crystals induced under polarized light}

Akira Hirano, Daichi Kitagawa and Seiya Kobatake*

\begin{abstract}
Materials that change in their shape by external stimuli have been attracting much attention as artificial mesoscopic actuators. Especially, photoresponsive molecular crystals are promising materials as photoactuators. Although many kinds of crystals exhibiting photomechanical motions have been reported so far, in many cases, light is irradiated to the crystal homogeneously. The details of the effect of irradiation conditions have not been specifically examined. Herein, the effect of polarized ultraviolet (UV) light on the photomechanical bending motion of diarylethene crystals was investigated. The bending speed upon polarized UV light depended on the polarization angle. The dependence of the bending speed was well correlated with the absorption anisotropy of the open-ring isomer in the crystal, which indicates that the photochromic reaction depth from the crystal surface played an important role in controlling the bending speed.
\end{abstract}

\section{Introduction}

Assemblies of aligned photoresponsive molecules lead to new functional photomechanical materials such as molecular machines, molecular rotors, molecular robots and artificial muscles. ${ }^{1,2}$ Photoresponsive organic molecular crystals and liquid crystalline elastomers are known as representative photomechanical materials. In particular, photoresponsive molecular crystals are the most promising materials in application to artificial microscopic photoactuators because of their rapid response, high fatigue resistance and high Young's modulus. ${ }^{3}$ Although various compounds that exhibit a photomechanical crystal deformation have been reported so far, such as furylfulgides, ${ }^{4}$ azobenzenes, ${ }^{5}$ salicylideneanilines, ${ }^{6}$ anthracene derivatives ${ }^{7-13}$ and others, ${ }^{14-18}$ photomechanical crystals composed of photochromic diarylethene derivatives have an advantage in their thermal stability of both open- and closed-ring isomers compared with other photochromic molecules, which enables to control their photomechanical motions at a desired time and a desired space.

The photomechanical motions such as contraction, ${ }^{19}$ expansion, ${ }^{20}$ bending, ${ }^{20-29}$ twisting, $^{30,31}$ fragmentation $^{32}$ and curling 9,33 have been reported so far. Recently, the mathematical analysis of the photomechanical motions has been reported. ${ }^{34-37}$ To find new photomechanical motions, many researchers made efforts to design and synthesize new compounds and to change crystal growth methods, whereas no one has focused on the irradiation conditions. If the

Department of Applied Chemistry, Graduate School of Engineering, Osaka City

University, 3-3-138 Sugimoto, Sumiyoshi-ku, Osaka 558-8585, Japan.

E-mail: kobatake@a-chem.eng.osaka-cu.ac.jp; Fax: +81 66605 2797;

Tel: +81666052797

+ Electronic Supplementary Information (ESI) available: Crystallographic data, photomechanical bending behavior, absorption anisotropy and spectrum of incident visible light (PDF). Movies for photomechanical bending behavior (Video S1-S4). See DOI: 10.1039/x0xx00000x photomechanical motion can be controlled by irradiation conditions, it would be a more convenient and useful way. From such a point of view, we have recently investigated the dependence of photomechanical bending behavior of diarylethene crystals on the irradiation waveleng $\operatorname{th}^{23}$ and the power of UV light. ${ }^{25}$ These results have provided information that the bending behavior can be interpreted in terms of a bimorph model for crystal composed of reactant and photoproduct and that the local strain caused by photoisomerization of diarylethene molecules makes a cumulative contribution to the bending behavior. Moreover, recently, we have reported the dependence of photoinduced twisting motion on illumination direction. ${ }^{31}$ These results have provided new knowledge for photomechanical crystal deformation and a possibility for utilization of irradiation condition to tune photomechanical crystal deformation.

In this paper, we report the dependence of the photoinduced bending motion of diarylethene crystals of 1,2bis(2-methyl-5-(4-(1-naphthoyloxymethyl)phenyl)-3-thienyl)perfluorocyclopentene (1a) and 1,2-bis(2-methyl-5-pmethoxyphenyl-3-thienyl)perfluorocyclopentene (2a) (Scheme 1) on the polarization angle of ultraviolet (UV) light, while crystals $1 \mathbf{a}$ and $\mathbf{2 a}$ have different molecular packings in the crystals. Upon irradiation with polarized UV light, the rod-like crystals of 1a and $2 \mathbf{a}$ showed the photomechanical bending as well as using non-polarized light. However, the bending speed depended on the polarization angle. We will discuss the mechanism of photomechanical bending behavior upon polarized UV light based on the photochromic reaction depth from the crystal surface. 


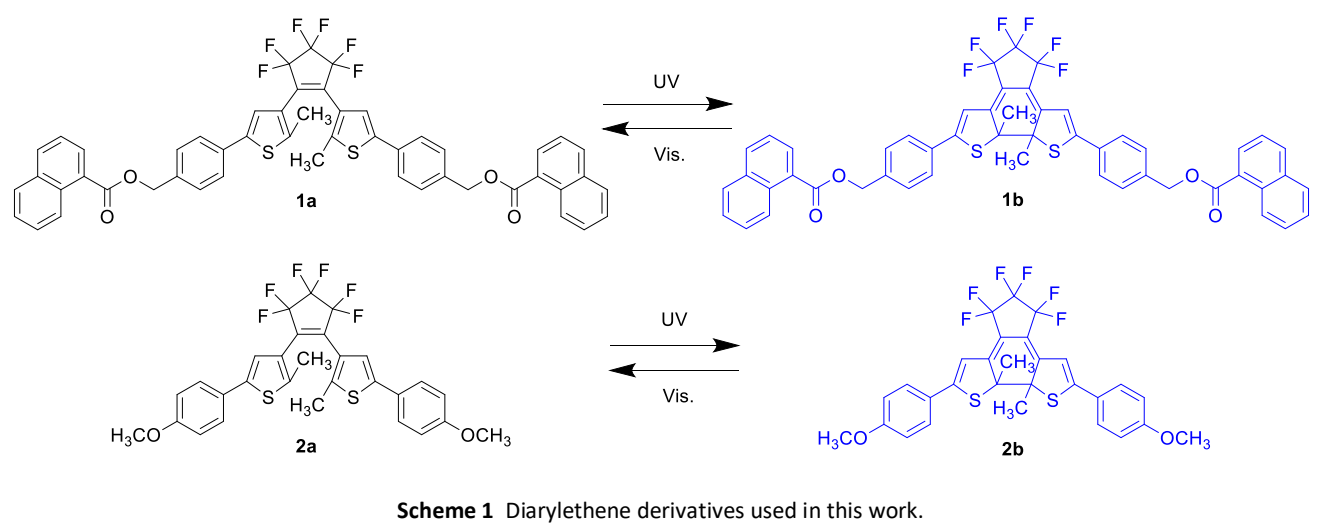

\section{Experimental}

\section{Photoirradiation}

UV irradiation was conducted using a Keyence UV-LED UV-400 attached with a UV-50H head ( $365 \mathrm{~nm}$ light). Polarized UV light irradiation was carried out through a Sigma Koki NSPFU-30C polarizer with a PH-30-ARS polarizer holder. The incident light intensity on the crystal surface was measured using a Neoark PM-335A power meter. The visible light was irradiated using a halogen lamp (100 W).

\section{Photoinduced crystal bending}

Photomechanical crystal deformation upon polarized UV light was observed as illustrated in Fig. 1. The polarized UV light was irradiated on (010) and (100) faces for crystals $1 \mathbf{a}$ and $\mathbf{2 a}$ respectively. The irradiation intensity of UV (365 nm) light through a polarizer was set to be constant at any angles of polarization. The edge of a rod-like crystal was fixed on thin glass, and a fluorescent material was painted on the thin glass. The fluorescence on the thin glass shows the onset time of UV irradiation. The photomechanical motion was observed using a Keyence VHX-500 digital microscope, and the frame rate was 28 fps. The 1 frame before the first frame with blue fluorescence on the glass was set to $0 \mathrm{~s}$. Therefore, the actual onset time is between 0 and $1 / 28 \mathrm{~s}$.

\section{Materials}

Diarylethenes $\mathbf{1 a}$ and $\mathbf{2 a}$ were synthesized according to a procedure previously described in the literature. ${ }^{20,38}$

\section{Results and discussion}

Photomechanical behavior under irradiation with non-polarized UV light

The rod-like crystals of $\mathbf{1} \mathbf{a}$ and $\mathbf{2 a}$ can be obtained by recrystallization from $n$-hexane/acetone and $n$-hexane, respectively. ${ }^{20,38}$ Rod-like crystal $1 \mathbf{a}$ always has a triclinic crystal system and a space group of $P \overline{1}$, and has (010), (0 $\overline{1} 0),(001)$ and $(00 \overline{1})$ faces. The $(010)$ and $(0 \overline{1} 0)$ faces are always welldeveloped in comparison with the (001) and (00 $\overline{1})$ faces. The molecules of 1a in the crystal are packed in the anti-parallel conformation which has sufficiently short distance to undergo the photocyclization reaction in the crystalline phase. ${ }^{39}$ The molecular packings of $\mathbf{1 a}$ are shown in Fig. 2a. The molecules are oriented in parallel when viewed from (010) face, and the long axes of all molecules are directed in $\theta=\mathrm{ca} .135^{\circ}$. In contrast, 2a has four polymorphic forms when recrystallized from $n$ hexane. ${ }^{38}$ Only $\delta$-crystal is a rod-like crystal with a crystal system of monoclinic $P 2_{1} / c$ and $Z=4$. Herein, we collected rodlike $\delta$-crystals to use as photomechanical materials. The rod-like $\delta$-crystal has (100), (101), (001), ( $\overline{1} 00),(\overline{1} 0 \overline{1})$ and $(00 \overline{1})$ faces.

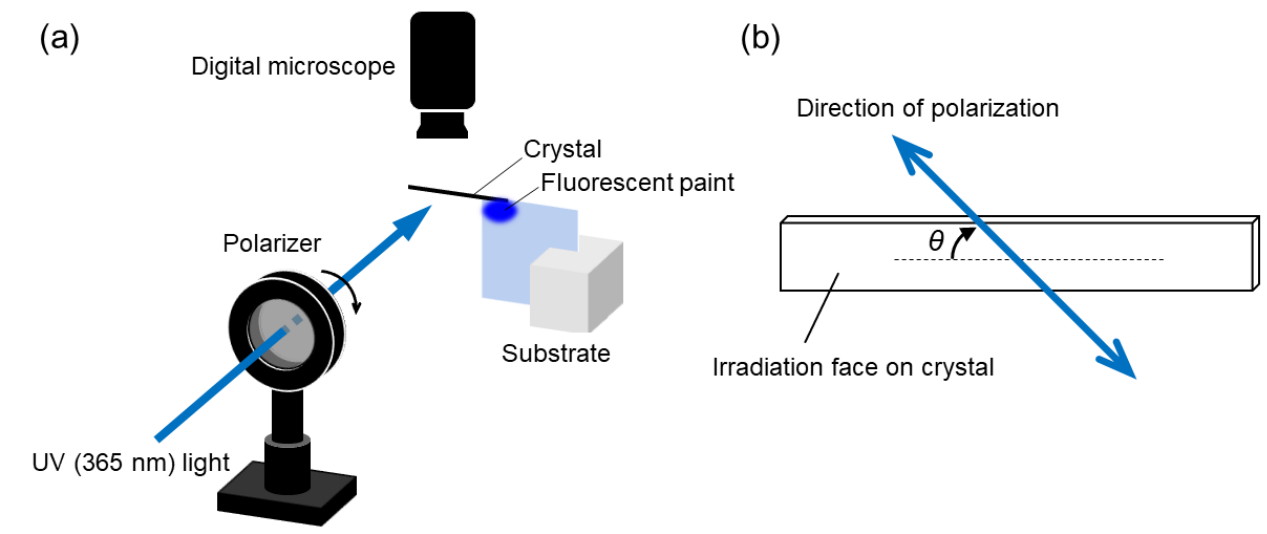

Fig. 1 (a) Experimental setup for photomechanical bending using polarized UV light and (b) definition of the angle of polarization 
(a)

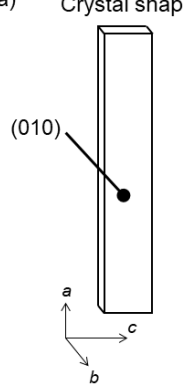

(b) Crystal shape

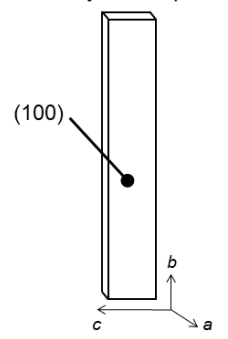

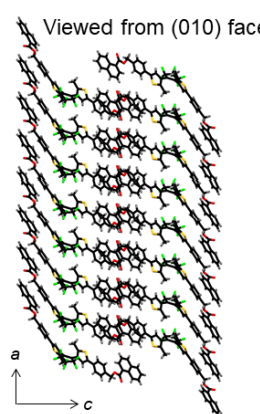

Viewed from (100) face

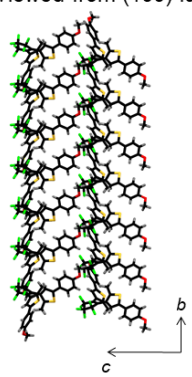

Fig. 2 Crystal shape and molecular packing of (a) $1 \mathbf{a}$ and (b) $\mathbf{2 a}$

The (100) and $(\overline{1} 00)$ faces are always well-developed. The molecular packings of crystal $\mathbf{2 a}$ are shown in Fig. $2 \mathrm{~b}$. The crystallographic data are summarized in Table S1.

Rod-like crystals bend away from the incident light or bend toward the incident light accompanied by the photochromic reaction when irradiated with UV light. ${ }^{25}$ The direction of the bending largely depends on the molecular packing in the crystal. When UV irradiation was conducted on the (010) face of crystal 1a, as shown in Fig. S1a, + the rod-like crystal of 1 a bent away from the incident UV light, and the bent crystal returned to the initial straight shape by visible light irradiation. The rod-like crystal of $\mathbf{2 a}$ bent toward the incident light when it was irradiated with UV light, as shown in Fig. S1b. + These bending behaviors are ascribed to the photochromic reaction only in the vicinity of the crystal surface and contraction or expansion to the long axis of the crystal in the reaction area.

\section{Effect of polarized UV light on photomechanical behavior}

The reaction depth from the crystal surface is one of the important factors to determine the bending speed. The use of polarized light in regularly aligned molecules such as crystals results in different absorption intensities in the crystal. Herein we investigated the effect of polarized UV light on the photomechanical crystal bending using crystal 1a. The crystal exhibited the photomechanical bending away from the light source upon irradiation with polarized UV light as well as that with non-polarized light. Interestingly, it was found that the bending speed depended on the polarization angle of UV light.

Fig. 3 shows the dependence of the bending speed on the polarization angle and snapshots of the bending behavior of crystal 1a. Here, the bending speed is defined as the curvature after UV irradiation for $1 \mathrm{sec}$. When the polarization angle was $0^{\circ}$ (i.e., the polarization direction was along with the long axis of the crystal), the bending speed was determined to be $0.60 \mathrm{~mm}^{-1}$ $\mathrm{s}^{-1}$. By rotating the polarization direction, the bending speed increased up to $1.1 \mathrm{~mm}^{-1} \mathrm{~s}^{-1}$ at $\theta=45^{\circ}\left(\theta_{\max }\right)$, decreased down to $0.19 \mathrm{~mm}^{-1} \mathrm{~s}^{-1}$ at $\theta=135^{\circ}\left(\theta_{\min }\right)$ and returned back to the initial speed at $\theta=180^{\circ}$. Thus, the bending speed cyclically changed against the polarization angle of UV light. Fig. 3b shows the polar plots of the bending speed upon polarized UV light. This result clearly revealed that the use of polarized UV light induces the bending speed anisotropy. Upon irradiation with non-polarized UV light which has the same irradiation power as the polarized light on the crystal surface, the bending speed was the average of those at $\theta_{\max }$ and $\theta_{\min }$ upon irradiation with polarized light. The direction at $\theta_{\max }$ depicted as the dashed arrow in Fig. $3 c$ is almost perpendicular to the long axis of $1 \mathrm{a}$ molecule. The relationship between this direction and absorption of 1a molecule in the crystal is discussed in the next section.

\section{Polarized absorption spectra of colorless crystal}

The photomechanical bending deformation requires the bimorph structure between the open-ring isomers and the photogenerated closed-ring isomers in the crystal surface. To reveal why the polarization angle affects the bending speed, it is necessary to clarify the absorption anisotropy of crystal $\mathbf{1 a}$. Thus, we measured polarized absorption spectra of crystal $\mathbf{1 a}$.
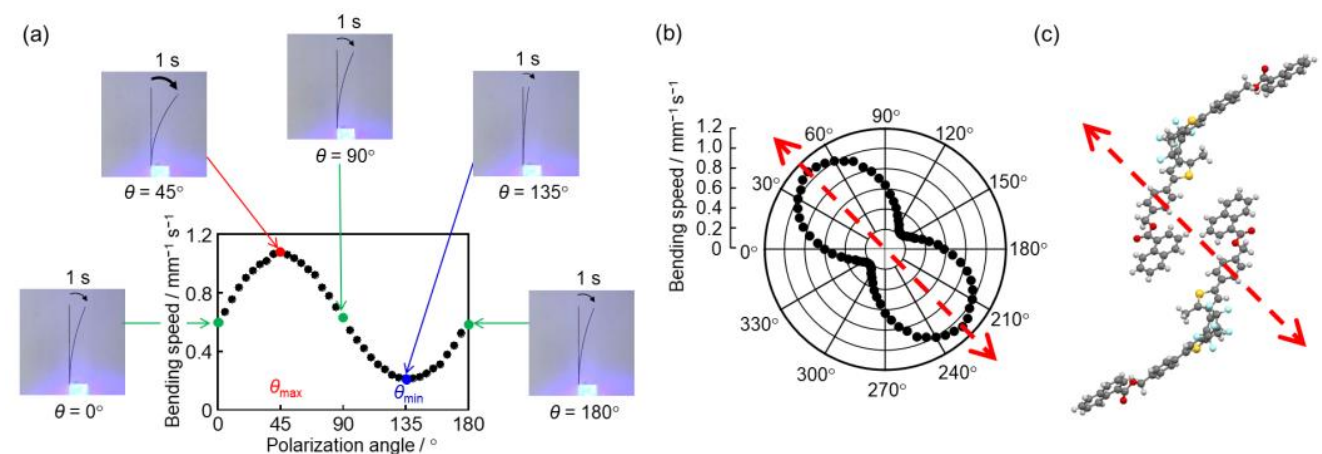

Fig. 3 (a) The dependence of the bending speed on the polarization angle and snapshots of the bending behavior of crystal 1a (thickness: $3.8 \mu \mathrm{m}$ ), (b) polar plots of the bending speed for crystal 1a and (c) the correlation between the molecular orientation and the direction of $\theta_{\text {max. }}$ Irradiation intensity of polarized UV light is $27.4 \mathrm{~mW} \mathrm{~cm}^{-2}$. The red dashed arrow shown in (c) indicates the direction of $\theta_{\max }$. 


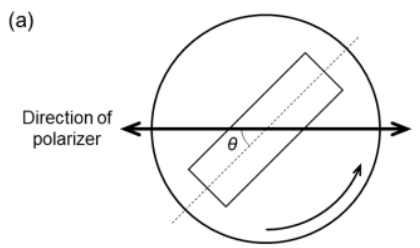

(b)

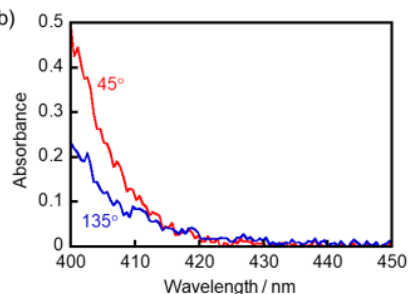

(c)

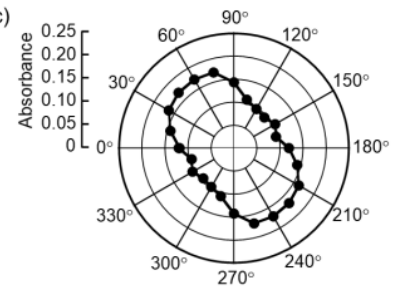

Fig. 4 (a) Definition of rotation angle $\theta$ for measurement of polarized absorption spectra, (b) polarized absorption spectra of crystal 1a and (c) polar plots of absorbance at $408 \mathrm{~nm}$ for crystal 1 a.
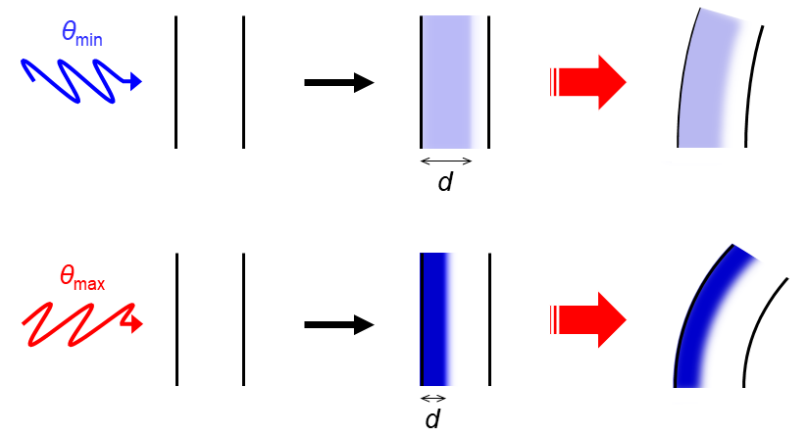

Fig. 5 Schematic illustration for mechanism of photomechanical bending behavior using polarized UV light with different polarization angles.
The absorption of 1a in the crystal at $365 \mathrm{~nm}$ is too large, thus the light does not penetrate the crystal. We measured the absorption at the absorption edge. Fig. 4 shows the polarized absorption spectra of $1 \mathbf{a}$ in the crystal. Note that the rotation angle $\theta$ in Fig. 4 is the same as the polarization angle $\theta$ in Fig. 1. The absorbance at the absorption edge was the largest when the crystal was rotated at $\theta=45^{\circ}$ corresponding to $\theta_{\max }$ in Fig. 3. The absorbance decreased as the crystal was rotated, and it became minimum at $\theta=135^{\circ}$ corresponding to $\theta_{\min }$ in Fig. 3. The absorption anisotropy of open-ring isomer in Fig. 4c was completely different from that of the closed-ring isomer in Fig. S2b. The polar plots for absorbance of $1 \mathbf{a}$ in the crystal reveal that there is a good correlation between the bending speed anisotropy and the absorption anisotropy of 1a. This result indicates that the bending speed is proportional to the absorbance of diarylethene molecules in the crystal.

\section{Mechanism}

The bending speed was correlated with the absorption of $1 \mathrm{a}$ in the crystal when the crystal was irradiated with polarized UV light. This result implies that the polarization angle of UV light changes the photochromic reaction depth from the crystal surface $(d)$ as shown in Fig. 5. ${ }^{20,23}$ At $\theta_{\max }$, the photocyclization reaction takes place only in the vicinity of the crystal surface because of high absorption. As a result, $d$ value would be small and the bending speed would be large. In contrast, at $\theta_{\min }$, the absorbance is relatively small and the photocyclization reaction takes place in deeper from the crystal surface, which leads to large $d$ value. As a result, the bending speed at $\theta_{\min }$ would be small.

To confirm the difference in $d$ induced by different angles of polarized UV light, we investigated the back behavior of the bent crystal with visible light. The spectrum of the visible light was shown in Fig. S3. + Fig. 6 shows the snapshots of the bending behavior in Video S1-S4, + and Fig. 7 shows the curvature change of crystal 1a (crystal thickness: $2.7 \mu \mathrm{m}$ ) upon irradiation with UV and visible light. Herein, when the crystal bends toward the right side on the image, the curvature is defined as a positive. When the visible light was irradiated from the right side (Fig. (a)

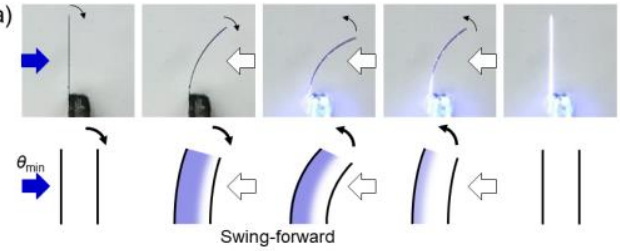

(c)

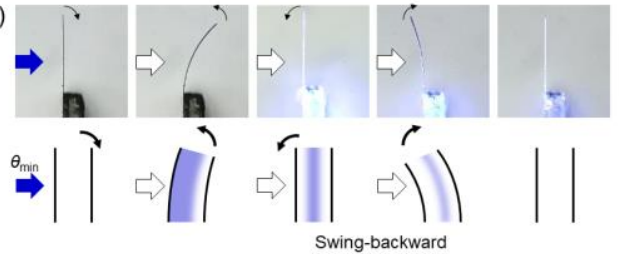

(b)
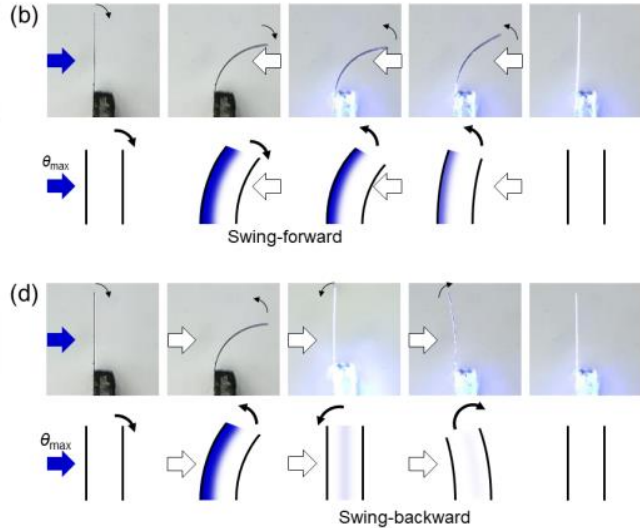

Fig. 6 Snapshots of bending behavior of 1a crystal (thickness: $2.7 \mu \mathrm{m}$ ) and illustration of the proposed mechanism. Polarized UV light irradiation was conducted from the left side on the image in all conditions. The angle of polarized UV light is $\theta_{\min }\left(a\right.$ and $c$ ) and $\theta_{\max }(b$ and $d)$. The visible light irradiation was carried out from the right side (a and $b$ ) and left side ( $c$ and d) on the image. Irradiation intensity of polarized UV light is $27.4 \mathrm{~mW} \mathrm{~cm}^{-2}$. 
(a)

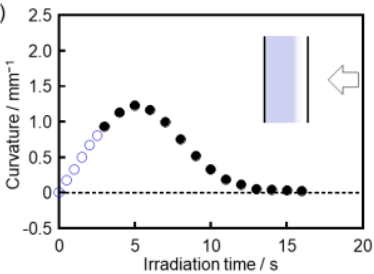

(c)
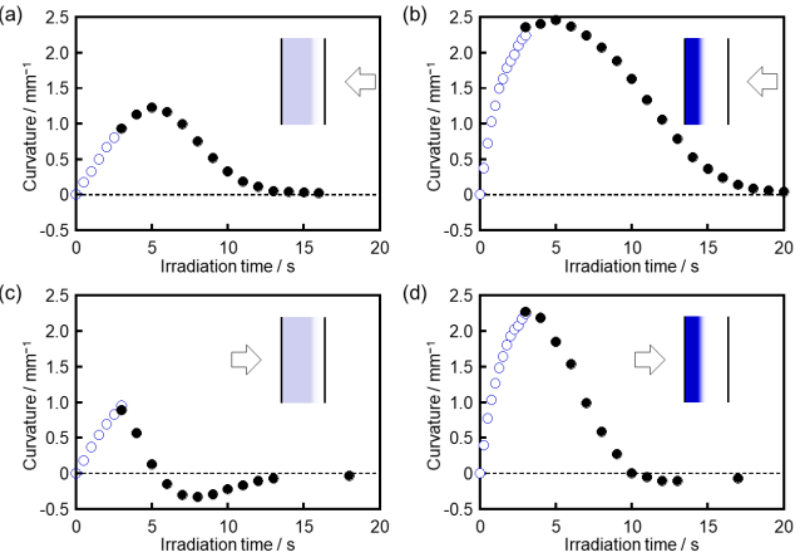

Fig. 7 Curvature change of crystal 1a (thickness: $2.7 \mu \mathrm{m}$ ) accompanied with the polarized UV light (blue open circle) and following visible light irradiation (black filled circle). The curvature was defined as a positive when the crystal bends toward the right side on the image. The angle of polarized UV light is $\theta_{\min }\left(a\right.$ and $c$ ) and $\theta_{\max }$ ( $b$ and $d$ ). The visible light irradiation was carried out from the right side ( $a$ and $b$ ) and left side (c and d). Irradiation intensity of polarized UV light is $27.4 \mathrm{~mW} \mathrm{~cm}-2$.

$6 a, b)$, the curvature increased at the initial stage of visible light irradiation. After that, the crystal returned to initial straight shape slowly. Such 'swing-forward' motion was observed remarkably when the UV light was irradiated at $\theta_{\min }$ (Fig. 6a). On the other hand, when the visible light was irradiated from the left side (Fig. 6c,d), the crystal returned to initial straight shape quickly and bent toward the visible light source going pass the initial straight shape. This 'swing-backward' motion was clearly observed when the UV light was irradiated at $\theta_{\min }$ (Fig. 6c). These unique behaviors can be explained by consideration of the difference in $d$, as mentioned above. The schematic illustrations of the proposed mechanism of the bending behaviors are also shown in Fig. 6 . When the visible light is irradiated from the right side, the right side of the crystal relatively shrinks due to the photocycloreversion reaction to result in more bending. Such change becomes larger when the reaction depth from the crystal surface is large, which means the case that UV light was irradiated at $\theta_{\min }$. As a result, the swing-forward motion was clearly observed at $\theta_{\min }$ compared to $\theta_{\text {max }}$. In the same way, the swing-backward motion can be

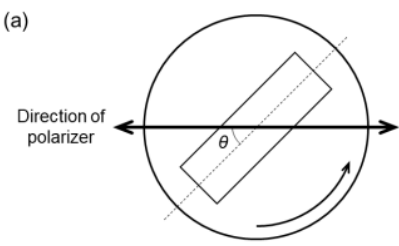

(b)

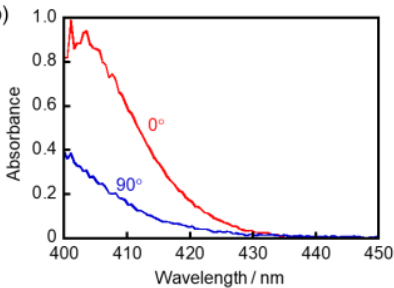

(c)

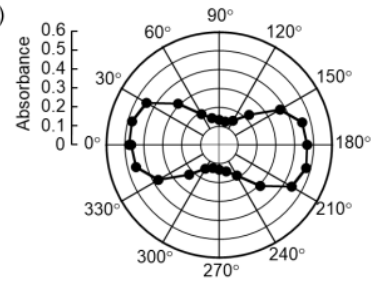

Fig. 9 (a) Definition of rotation angle $\theta$ for measurement of polarized absorption spectra, (b) polarized absorption spectra of crystal $\mathbf{2 a}$ and (c) polar plots of absorbance at $413 \mathrm{~nm}$ for crystal $\mathbf{2 a}$.

explained. When visible light is irradiated from the left side, the closed-ring isomer turns into the open-ring isomer. The photocycloreversion reaction proceeds from the surface and the left side shrinks compared to right side. As a result, the crystal bends toward the visible light source due to the existence of the closed-ring isomers in the middle part of the crystal.

\section{Universality}

To confirm the universality of the effect of polarized UV light on photomechanical bending motion of diarylethene crystals, we investigated the dependence of bending speed on the polarization angle using crystal $\mathbf{2 a}$. The types of molecular orientation viewed from the irradiation face are different in crystals $\mathbf{1 a}$ and $\mathbf{2 a}$, as shown in Fig. 2. Each molecule is packed in parallel in crystal 1a, whereas molecules are packed in almost perpendicular to each other in crystal 2a. UV irradiation was

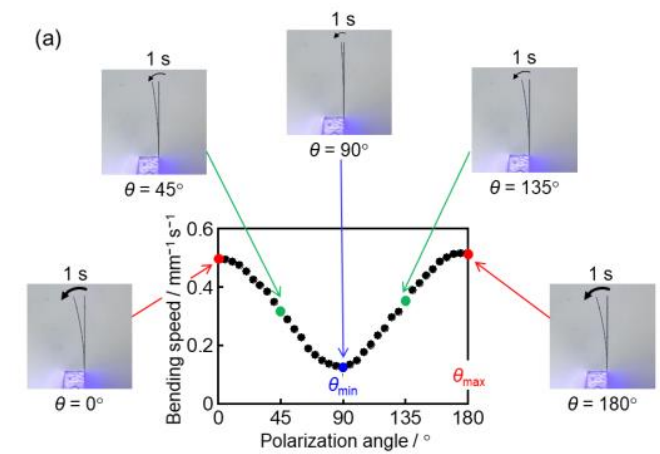

(b)

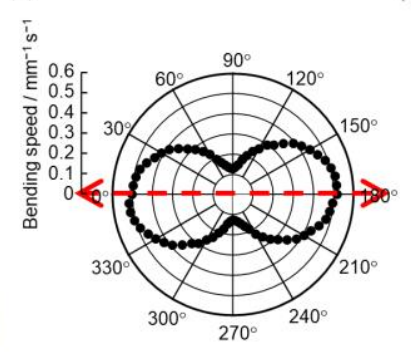

(c)

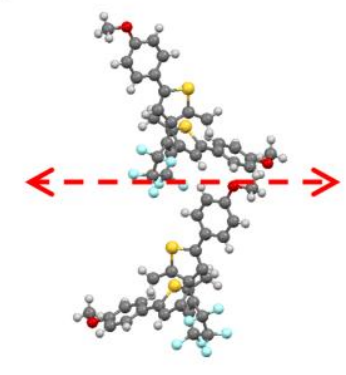

Fig. 8 (a) The dependence of the bending speed on the polarization angle and snapshots of the bending behavior of crystal $2 \mathrm{a}$ (thickness: $2.5 \mu \mathrm{m}$ ), (b) polar plots of the bending speed for crystal $2 \mathrm{a}$ and (c) the correlation between the molecular orientation and the direction of $\theta_{\text {max. }}$. Irradiation intensity of polarized UV light is $6.7 \mathrm{~mW} \mathrm{~cm}^{-2}$. The red arrow shown in (c) indicate the direction of $\theta_{\max }$. 
conducted on the (100) face of crystal $2 \mathbf{a}$. The crystal of $2 \mathbf{a}$ bent toward the incident light, as shown in Fig. S1b. † Fig. 8 shows the dependence of the bending speed on the polarization angle and snapshots of the bending behavior of crystal $2 \mathbf{a}$. The bending speed for crystal $\mathbf{2 a}$ also depended on the polarization direction as shown in Fig. $8 \mathrm{a}$. In the case of crystal $\mathbf{2 a}$, the bending speed was the largest value with $0.50 \mathrm{~mm}^{-1} \mathrm{~s}^{-1}$ at $0^{\circ}\left(\theta_{\max }\right)$, where the polarization direction was parallel to the long axis of the crystal. By rotating the polarization direction, the bending speed decreased, reaching $0.13 \mathrm{~mm}^{-1} \mathrm{~s}^{-1}$ at $90^{\circ}\left(\theta_{\mathrm{min}}\right)$. The bending speed anisotropy was also observed in crystal $\mathbf{2 a}$, and we measured the polarized absorption spectra of crystal $\mathbf{2} \mathbf{a}$ to know the absorption anisotropy. It was reported that the closed-ring isomer $\mathbf{2} \mathbf{b}$ in the crystal of $\mathbf{2} \mathbf{a}$ does not show the absorption anisotropy as shown in Fig. S2ct because the molecules are packed in the herringbone type molecular packing. ${ }^{38}$ On the other hand, we found that the open-ring isomer $\mathbf{2} \mathbf{a}$ in the crystal exhibits the absorption anisotropy, as shown in Fig. 9. The polar plots of the absorbance at $413 \mathrm{~nm}$ in crystal $2 \mathrm{a}$ indicate that there is a good correlation between the bending speed anisotropy and the absorption anisotropy of the crystal. The absorption anisotropy of $\mathbf{2 a}$ in the crystal is different from that of 1a in the crystal. These differences in absorption anisotropy are due to the difference in the molecular orientation between crystals $\mathbf{1 a}$ and $\mathbf{2 a}$. What is common to $\mathbf{1 a}$ and $\mathbf{2 a}$ is that the absorption anisotropy is different between open- and closed-ring isomer and the bending speed is proportional to the absorbance of diarylethene open-ring isomers in the crystal.

\section{Conclusions}

We have investigated the photoinduced bending behavior of rod-like crystals upon irradiation with polarized UV light. We demonstrated that the bending speed anisotropy was well correlated to the absorption anisotropy of diarylethene crystals, which indicates that the photochromic reaction depth from the crystal surface plays an important role in the photomechanical crystal bending. The unique bending behavior such as 'swingforward' and 'swing-backward' motions induced by polarized UV and visible light irradiation could be well explained by considering the reaction depth from the crystal surface. These results would provide not only the potential use of polarized light to tune photomechanical crystal deformations but also an advantage of changing the irradiation conditions to control the photomechanical material deformations.

\section{Conflicts of interest}

There are no conflicts to declare.

\section{Acknowledgements}

This work was partly supported by JSPS KAKENHI Grant Number JP26107013 in Scientific Research on Innovative Areas "Photosynergetics" to S.K. and JSPS KAKENHI Grant Number
JP16K17896 in Scientific Research for Young Scientists (B) to D.K. The authors also thank Nippon Zeon Co., Ltd. for providing octafluorocyclopentene.

\section{Notes and references}

1 J. M. Abendroth, O. S. Bushuyev, P. S. Weiss and C. J. Barrett, ACS Nano, 2015, 9, 7746-7768.

2 A. Priimagi, C. J. Barrett and A. Shishido, J. Mater. Chem. C, 2014, 2, 7155-7162.

3 M. Irie, T. Fukaminato, K. Matsuda and S. Kobatake, Chem. Rev., 2014, 114, 12174-12277.

4 H. Koshima, H. Nakaya, H. Uchimoto and N. Ojima, Chem. Lett., 2012, 41, 107-109.

5 H. Koshima, N. Ojima and H. Uchimoto, J. Am. Chem. Soc., 2009, 131, 6890-6891.

6 H. Koshima, K. Takeuchi, H. Uchimoto, M. Shiro and D. Hashizume, Chem. Commun., 2011, 47, 11423.

7 R. O. Al-Kaysi, A. M. Müller and C. J. Bardeen, J. Am. Chem. Soc., 2006, 128, 15938-15939.

8 R. O. Al-Kaysi and C. J. Bardeen, Adv. Mater., 2007, 19, 12761280.

9 T. Kim, M. K. Al-Muhanna, S. D. Al-Suwaidan, R. O. Al-Kaysi and C. J. Bardeen, Angew. Chem. Int. Ed., 2013, 52, 6889-6893.

10 L. Zhu, R. O. Al-Kaysi, R. J. Dillon, F. S. Tham and C. J. Bardeen, Cryst. Growth Des., 2011, 11, 4975-4983.

11 L. Zhu, R. O. Al-Kaysi and C. J. Bardeen, J. Am. Chem. Soc., 2011 133, 12569-12575.

12 L. Zhu, R. O. Al-Kaysi and C. J. Bardeen, Angew. Chem. Int. Ed., 2016, 55, 7073-7076.

13 F. Tong, M. Al-Haidar, L. Zhu, R. O. Al-Kaysi, C. J. Bardeen, Chem. Commun., 2019, in press [DOI: 10.1039/C8CC10051A].

14 K.-J. Chen, Y.-C. Thai, Y. Suzuki, K. Osakada, A. Miura and M. Horie, Nature Commun., 2016, 7, 13321.

15 T. Seki, K. Sakurada, M. Muromoto and H. Ito, Chem. Sci., 2015, 6, 1491-1497.

16 H. Nakai, K. Matsuba, M. Akimoto, T. Nozaki, T. Matsumoto, K. Isobe, M. Irie and S. Ogo, Chem. Commun., 2016, 52, 43494352.

17 J.-K. Sun, W. Li, C. Chen, C.-X. Ren, D.-M. Pan and J. Zhang, Angew. Chem. Int. Ed., 2013, 52, 6653-6657.

18 Y. Zhang, C. Peng, Z. Zhou, R. Duan, H. Ji, Y. Che, J. Zhao, Adv. Mater., 2015, 27, 320-325.

19 S. Kobatake, S. Takami, H. Muto, T. Ishikawa and M. Irie, Nature, 2007, 446, 778-781.

20 D. Kitagawa and S. Kobatake, J. Phys. Chem. C, 2013, 117, 20887-20892.

21 D. Kitagawa and S. Kobatake, Photochem. Photobiol. Sci., 2014 13, 764 .

22 D. Kitagawa, C. Iwaihara, H. Nishi and S. Kobatake, Crystals, 2015, 5, 551-561.

23 D. Kitagawa, R. Tanaka and S. Kobatake, Phys. Chem. Chem. Phys., 2015, 17, 27300-27305.

24 D. Kitagawa and S. Kobatake, Chem. Commun., 2015, 51, 4421-4424.

25 A. Hirano, T. Hashimoto, D. Kitagawa, K. Kono and S. Kobatake, Cryst. Growth Des., 2017, 17, 4819-4825.

26 K. Uchida, S. Sukata, Y. Matsuzawa, M. Akazawa, J. J. D. de Jong, N. Katsonis, Y. Kojima, S. Nakamura, J. Areephong, A. Meetsma and B. L. Feringa, Chem. Commun., 2008, 326-328.

27 M. Morimoto and M. Irie, J. Am. Chem. Soc., 2010, 132, 14172-14178.

28 S. Ohshima, M. Morimoto and M. Irie, Chem. Sci., 2015, 6, 5746-5752.

29 F. Terao, M. Morimoto and M. Irie, Angew. Chem. Int. Ed., 2012, 51, 901-904. 
30 D. Kitagawa, H. Nishi and S. Kobatake, Angew. Chem. Int. Ed., 2013, 52, 9320-9322.

31 D. Kitagawa, H. Tsujioka, F. Tong, X. Dong, C. J. Bardeen and S. Kobatake, J. Am. Chem. Soc., 2018, 140, 4208-4212.

32 D. Kitagawa, T. Okuyama, R. Tanaka and S. Kobatake, Chem. Mater., 2016, 28, 4889-4892.

33 H. Wang, P. Chen, Z. Wu, J. Zhao, J. Sun and R. Lu, Angew. Chem. Int. Ed., 2017, 56, 9463-9467.

34 N. K. Nath, L. Pejov, S. M. Nichols, C. Hu, N. Saleh, B. Kahr, P. Naumov, J. Am. Chem. Soc., 2014, 136, 2757-2766.

35 P. Naumov, S. Chizhik, M. K. Panda, N. K. Nath and E. Boldyreva, Chem. Rev., 2015, 115, 12440-12490.

36 S. Chizhik, A. Sidelnikov, B. Zakharov, P. Naumov and E. Boldyreva, Chem. Sci., 2018, 9, 2319-2335.

37 M. Kim, J.-H. Yun and M. Cho, Sci. Rep., 2017, 7, 967.

38 M. Morimoto, S. Kobatake and M. Irie, Chem.-Eur. J., 2003, 9, 621-627.

39 S. Kobatake, K. Uchida, E. Tsuchida and M. Irie, Chem. Commun., 2002, 2804-2805. 